\title{
Influence of Crystal Quality on the Excitation and Propagation of Surface and Bulk Acoustic Waves in Polycrystalline AIN Films
}

\author{
Marta Clement, Jimena Olivares, Jose Capilla, Jesús Sangrador, and Enrique Iborra
}

\begin{abstract}
We investigate the excitation and propagation of acoustic waves in polycrystalline aluminum nitride films along the directions parallel and normal to the $c$-axis. Longitudinal and transverse propagations are assessed through the frequency response of surface acoustic wave and bulk acoustic wave devices fabricated on films of different crystal qualities. The crystalline properties significantly affect the electromechanical coupling factors and acoustic properties of the piezoelectric layers. The presence of misoriented grains produces an overall decrease of the piezoelectric activity, degrading more severely the excitation and propagation of waves traveling transversally to the $c$-axis. It is suggested that the presence of such crystalline defects in $c$-axis-oriented films reduces the mechanical coherence between grains and hinders the transverse deformation of the film when the electric field is applied parallel to the surface.
\end{abstract}

\section{INTRODUCTION}

$\mathrm{P}$ OLYCRYSTALLINE sputtered AIN films are currently used as piezoelectric active layers in RF filters based on BAW [1] and SAW devices [2], as well as in piezoelectrically actuated micro electromechanical systems (MEMS), such as gravimetric sensors [3], RF switches [4], or mechanical resonators [5]. Under controlled deposition conditions, AlN sputtered films exhibiting a wurtzite structure tend to grow with a definite preferred $c$-axis orientation, which means that the vast majority of the microcrystals grow with the $\{00 \cdot 2\}$ planes parallel to the surface of the film [6]. However, depending on the deposition conditions, the geometrical setup, the nature and roughness of the substrate, or the residual gases in the deposition chamber [7]-[9], grains exhibiting other orientations, such as (10.1), $(10 \cdot 2)$ and (10.3), may also be present. The existence of such misaligned grains is usually accompanied by other structural defects that significantly reduce the piezoelectric activity of the films, very likely because they stimulate the growth of grains with polarization opposite to the dominant polarization, which cancels part of the piezo-

Manuscript received June 6, 2011; accepted November 8, 2011. This work was supported by the Ministerio de Ciencia e Innovación of Spain through project MAT2010-18933, by the Comunidad de Madrid and the Universidad Politécnica de Madrid through the IV Plan Regional de Investigación Científica e Innovación Tecnológica (PRICIT) program, and by the European Union through the European Regional Development Fund (FEDER).

The authors are with the Grupo de Microsistemas y Materiales Electrónicos del Centro de Materiales y Dispositivos Avanzados para las TIC (GMME-CEMDATIC), Universidad Politécnica de Madrid (e-mail: mclement@etsit.upm.es).

Digital Object Identifier 10.1109/TUFFC.2012.2163 electric response [10]. Additionally, highly c-axis-textured films with poor piezoelectric activity containing grains with opposite polar orientations have also been reported [11]. Thus, the overall piezoelectric activity in polycrystalline films must be considered as the combination of the individual responses of the different grains $[6]$ and is, hence, influenced by the crystal quality of the film, the polar orientation of the microcrystals, and, of course, by the intergrain regions in the film.

The driving of the piezoelectric AlN films used in acoustic structures (SAW and BAW) requires electric fields either parallel or perpendicular to the $c$-axis, which, by virtue of the inverse piezoelectric effect, produce the desired deformation that is propagated. Although the piezoelectric coefficients $\left(d_{i j}\right)$ relating the electric field and the strain in the different directions have well-defined values in monocrystalline films, the piezoelectric properties of polycrystalline films are affected by the crystalline characteristics of the film. In practical SAW and BAW devices, the magnitude used to assess the effectiveness of energy conversion from electrical to mechanical is the effective electromechanical coupling coefficient $\left(k_{\text {eff }}^{2}\right)$, defined as the ratio between the mechanical power generated and the applied electrical power at the resonance. This coupling factor depends undoubtedly on the physical properties of the polycrystalline AIN layer, but also on other external parameters, such as the device's mechanical configuration (mechanical isolation and losses) and the electrical parasitics (resistance of electrodes or dielectric losses of insulators) [12], [13]. The use of physical models capable of simulating the electrical frequency response of SAW and BAW devices [14], [15] allows successful separation of the contribution of the parasitics and geometrical effects, which provides a ideal coupling coefficient dependent only on the physical properties (elastic, dielectric, and piezoelectric) of the piezoelectric films. This kind of analysis, permitting independent assessment of the different contributions to the overall response of acoustic devices, is very convenient for optimizing both active material and device design.

In this paper, we investigate the excitation and propagation of acoustic waves in SAW and BAW devices as a function of the piezoelectric and crystalline properties of polycrystalline AIN films. The choice of these two kinds of devices is based on the belief that the anisotropic nature of the AlN polycrystalline films and presence of defects may differently affect the excitation and propagation of acoustic waves traveling in the plane of the film (Rayleigh 
SAWs) and those traveling normally to the film (longitudinal BAWs). To perform a reliable comparison, AlN films used in SAW and BAW devices were grown in the same run and on identical substrates to guarantee that the films exhibited equal crystalline and piezoelectric properties. Likewise, special care has been taken to guarantee that all SAW devices were composed of an identical set of layers (AIN and underlying layers of identical thickness), so that the crystal quality of the AlN film is the only characteristic that has been varied. The influence of the crystal orientation, grain size, and crystalline defects on the excitation and propagation of longitudinal and transverse acoustic waves is discussed.

\section{Experimental Techniques}

The substrates for BAW and SAW devices consisted of silicon wafers covered with a low-density silicon oxide layer, which was used as a simple acoustic mirror to confine the BAWs, coated with a 100-nm-thick sputtered Ir film used as the bottom electrode of the BAW resonators. The presence of the Ir layer was not required for SAW devices; moreover, it distorted the frequency response of the delay lines. However, it was kept to guarantee that the AlN films under study possessed identical crystalline structure, independent of the kind of device (SAW or BAW), because the crystalline properties of AlN films are strongly affected by the nature of the underlying substrate. AlN films of $1400 \mathrm{~nm}$ thickness were then deposited by pulsed direct current magnetron sputtering under different deposition conditions to vary their crystal quality: the voltage bias of the substrate was varied from -30 to $-80 \mathrm{~V}$ by applying a variable-power $\mathrm{RF}$ signal and the total pressure was varied from 1.5 to 5 mTorr; the concentration of $\mathrm{N}_{2}$ in $\mathrm{Ar}$ was kept at $70 \%$. Other process parameters, such as the time and the voltage of the substrate pre-etching, the total power, and the substrate temperature were also varied to achieve AIN films with different characteristics. The BAW and SAW devices were then completed by sputtering a 200-nm-thick Mo film that was patterned to define the top electrodes of the BAW resonators and the interdigital transducers (IDTs) of the SAW delay lines. The targeted frequencies were approximately $2 \mathrm{GHz}$ for the $\mathrm{BAW}$ resonators and $120 \mathrm{MHz}$ (wavelength of $40 \mu \mathrm{m}$ ) for the SAW delay lines.

The structure of the different AlN films was assessed by X-ray diffraction (XRD) measurements, infrared (IR) absorption spectroscopy in the reflectance mode, and atomic force microscopy (AFM). All of the details for AIN characterization are described in a previous work [10]. BAW resonators were characterized by measuring the electrical reflection coefficient $\left(S_{11}\right)$ at frequencies ranging from $100 \mathrm{kHz}$ to $3 \mathrm{GHz}$, using a network analyzer (PNA N5230A, Agilent Technologies Inc., Santa Clara, CA) connected to the samples through a Picoprobe coplanar RF probe (GBB Industries Inc., Naples, FL). The data were fitted to Mason's physical model [14] to derive the electromechanical cou- pling factor of an ideal resonator operating in the longitudinal thickness mode, which we will call $k_{\mathrm{BAW}}^{2}$. SAW delay lines were stuck to a test fixture and the pads of the IDTs were ultrasonically bonded to the test fixture using $25-\mu \mathrm{m}$ wide aluminum wires. Their scattering parameters, $S_{i j}$, were measured between $100 \mathrm{kHz}$ and $700 \mathrm{MHz}$ with the same network analyzer mentioned before. The experimental spectra were fitted using our own simulation program based on Campbell's method [15], which has been described in a previous work [16]. This fitting allowed elimination of all the parasitic effects that distort the frequency response of a real delay line, such as wiring effects (which add an inductive impedance to the ground loop), substrate effects (electromagnetic feedthrough resulting from the conductive character of the Si substrate and the underlying Ir layer), or the effects related to the thicknesses of the layered structure $\left(\mathrm{AlN} / \mathrm{Ir} / \mathrm{SiO}_{2}\right)$. After the fitting, we obtained the electromechanical coupling factor associated with the excitation of a Rayleigh SAW in an ideal semi-infinite AlN substrate, which we will call $k_{\mathrm{SAW}}^{2}$, only dependent on the AIN material properties and independent of the geometry of the layered structure. The experimental resonant frequency also allowed derivation of the acoustic velocity of the SAW $v_{\mathrm{SAW}}$ in the layered structure. The frequency response of a BAW resonator and a SAW delay line fabricated with identical AlN films is shown in Fig. 1. No attempt was made to optimize the performance of the devices (effective coupling factor and quality factor), because their only purpose was to assess the piezoelectric activity of the AlN films.

\section{Particle Displacement and \\ ELECTROMECHANICAL COUPLING IN BAW AND SAW DEVICES}

The electromechanical coupling factor $k^{2}$ in electrically driven piezoelectric devices is defined as the ratio between the stored mechanical energy and the input electrical energy. It can be expressed as

$$
k^{2}=\frac{d^{2} \cdot c}{\varepsilon_{0} \cdot \varepsilon}
$$

where $\varepsilon$ is the relative dielectric permittivity of the piezoelectric material, $c$ is the elastic constant, and $d$ is the piezoelectric constant. The variables of this general relationship are vectors and tensors, so the expression must be particularized for the different components of the electric fields, deformations, and material constants.

The BAW resonators assessed in this work consist of a $c$-axis-oriented AIN film sandwiched between two metallic electrodes that excite a longitudinal wave which propagates parallel to the $c$-axis, as shown in Figs. 2(a) and 2 (c). The electromechanical coupling factor, $k_{\mathrm{BAW}}^{2}$, of such structures can be written as [17]

$$
k_{\mathrm{BAW}}^{2}=\frac{d_{33}^{2} \cdot c_{33}^{\mathrm{D}}}{\varepsilon_{0} \cdot \varepsilon_{33}^{\mathrm{S}}}
$$




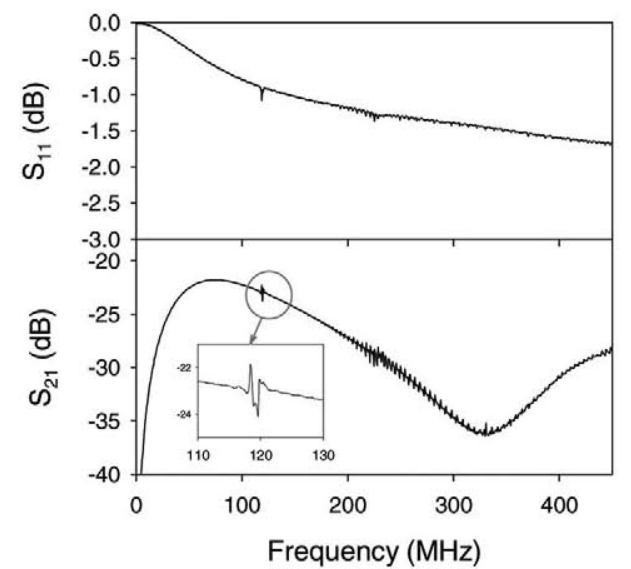

(a)

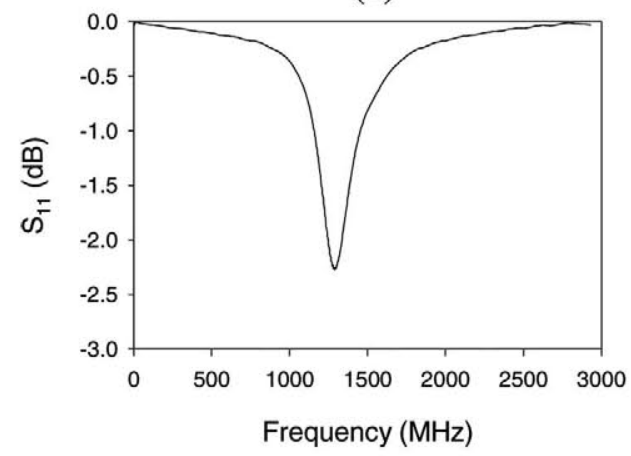

(b)

Fig. 1. Frequency response of (a) a SAW delay line and (b) a BAW resonator made with AlN films of high piezoelectric activity. After fitting the data, the values of $k_{\mathrm{SAW}}^{2}$ and $k_{\mathrm{BAW}}^{2}$ are $1.2 \%$ and $6.4 \%$, respectively.

where $d_{33}, c_{33}^{\mathrm{D}}$, and $\varepsilon_{33}^{\mathrm{S}}$ are the piezoelectric constant, the elastic constant at constant electric displacement, and the electric permittivity at constant strain, respectively, in the c-axis direction.

On the other hand, in the SAW devices investigated [see Fig. 2(b)], the exited Rayleigh wave propagating par-

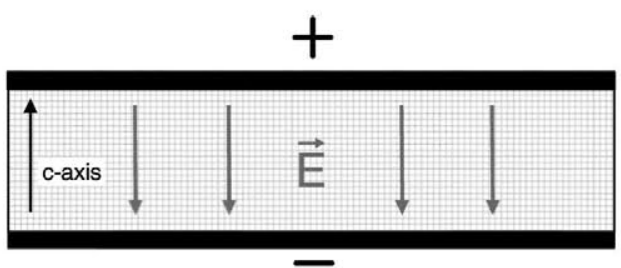

(a)

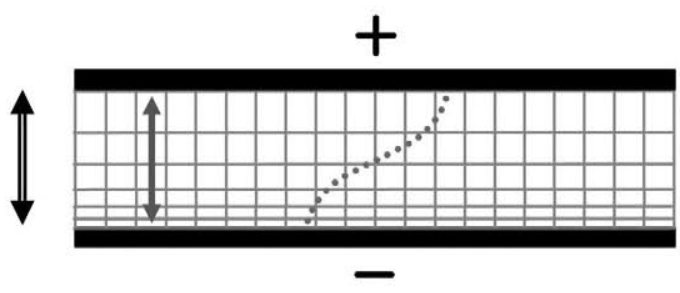

(c) allel to the surface involves both a combination of shearing and extensional motions [17]. Indeed, between the IDTs, the field lies on the film plane perpendicular to the polar axis; the resulting shear strains resulting from $d_{15}$ add to the strains of $d_{33}$ and $d_{31}$ below the electrodes, as shown in Fig. 2(d) [17], [18]. The relation between the coupling coefficient $k_{\text {SAW }}^{2}$ and the piezoelectric coefficients is already given by (1) but particularizing it for the electric field and deformations generated in the different directions is not straightforward. Additionally, in our layered structures, the effective coupling coefficient $k_{\text {SAW-eff }}^{2}$ also depends on the film thickness-to-wavelength ratio and the position of the transducer electrode (substrate-film boundary or at the upper film surface). The highest effective coupling efficiency in layered structures is achieved at film thicknessto-wavelength ratios of one-half [15], in which case, the main contribution comes from the $d_{15}$ piezoelectric constant [19]. In this work, the thickness of the AIN films $(1.4 \mu \mathrm{m})$ was considerably smaller than the wavelength of the acoustic wave $(40 \mu \mathrm{m})$; for this thickness-to-wavelength ratio, the effective coupling factor is very likely dominated by $d_{13}[19]$.

\section{Structural and Piezoelectric Characterization of AlN Films}

AlN films exhibiting different crystal qualities were subjected to a full structural, morphological, and piezoelectric characterization, which included the determination of the corresponding $k_{\mathrm{SAW}}^{2}$ and $k_{\mathrm{BAW}}^{2}$ through the analysis of the frequency response of SAW and BAW devices fabricated with them.

Fig. 3 shows the XRD patterns of four representative AlN films. The films range from perfectly $c$-axis-oriented, characterized by a single $(00 \cdot 2)$ reflection, to films exhibiting a clear $c$-axis orientation but additionally containing

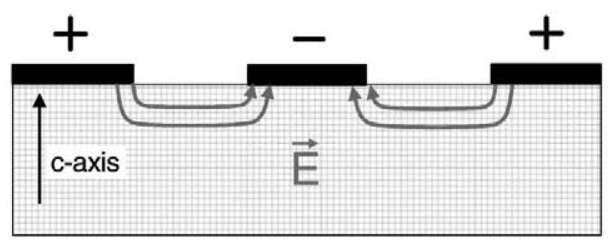

(b)

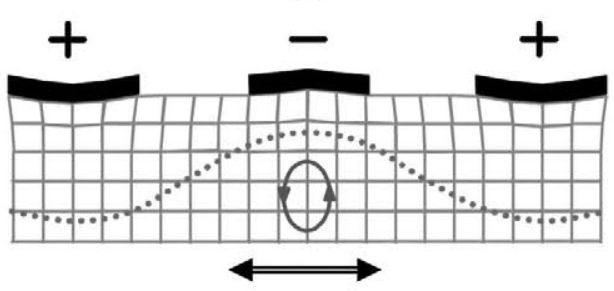

(d)

Fig. 2. Electrodes for the driving of the piezoelectric films in (a) BAW and (b) SAW devices and the corresponding electric fields. Schematic drawing of strains induced (c) by $d_{33}$ in BAW devices, and (d) by $d_{33}, d_{31}$, and $d_{15}$ below and between the electrodes in SAW devices. The pictures also show the acoustic waves (dotted lines), their direction of propagation (double arrows), and the particle movement (single arrows). 


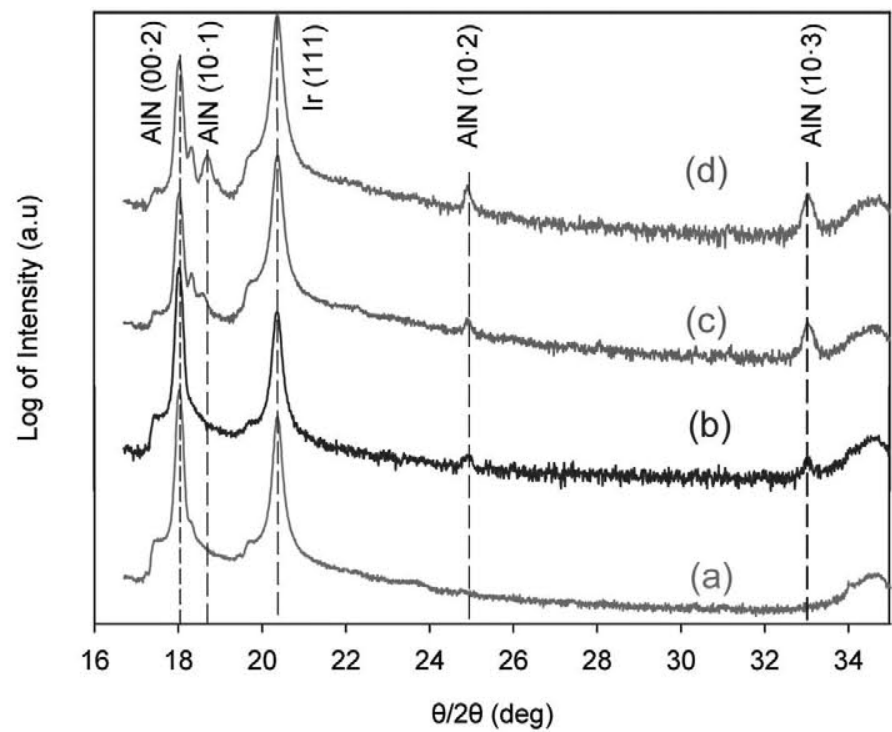

Fig. 3. X-ray diffraction (XRD) patterns of AlN films of different crystal quality. The XRD reflections are depicted in logarithmic scale to emphasize the weaker reflections.

different amounts of grains oriented in the (10.1), (10.2), and $(10 \cdot 3)$ directions. It is important to remember that the intensity of the $(10 \cdot 1),(10 \cdot 2)$, and $(10 \cdot 3)$ reflections is not directly related to the real amount of grains with those orientations; their low intensity can be explained by the strict geometrical condition imposed by the type of XRD experiment carried out $(\theta / 2 \theta$ scan $)[10]$. Indeed, whereas reflections with the $\{00 \cdot 2\}$ planes are observed regardless of the basal orientation of the (00.2)-oriented grains, only specific basal orientations of the tilted grains give $\{10 \cdot 1\}$, $\{10 \cdot 2\}$, or $\{10 \cdot 3\}$ planes parallel to the surface. These weak peaks may actually correspond to a large amount of misaligned grains. We have also observed that AIN films containing misaligned grains usually have wide rocking curves $(\mathrm{RCs})$ around the $(00 \cdot 2)$-AlN reflection, although these two defects do not always appear together. Under some deposition conditions, AlN films with narrow RCs exhibiting traces of $(10 \cdot 1),(10 \cdot 2)$, and (10.3) reflections can be achieved; contrarily, AlN films with a single $(00 \cdot 2)$ reflection but RC with a full-width at half-maximum (FWHM) as high as $10^{\circ}$ have also been deposited. Clear evidence of the presence of inclined grains is provided by the IR absorption measurements shown in Fig. 4.

In the hexagonal AlN wurtzite structure, two vibrational modes are infrared active: the A1 mode, associated with the $\mathrm{N}-\mathrm{Al}$ bonds parallel to the $c$-axis, and the E1 mode, related to the Al-N bonds lying in the basal plane of the wurtzite structure [20], [21]. Because these two modes are polar, they split into longitudinal optical (LO) and transverse optical (TO) components, which appear at around 900 and $660 \mathrm{~cm}^{-1}$, respectively. According to Snell's law of diffraction, and considering the directions of the bonds related to the $\mathrm{A} 1$ and $\mathrm{E} 1$ modes, under normal incidence the reflectance spectra of AlN films containing only $(00 \cdot 2)$-oriented grains exhibit a single A1(LO) mode, whereas films containing tilted grains show, addi-

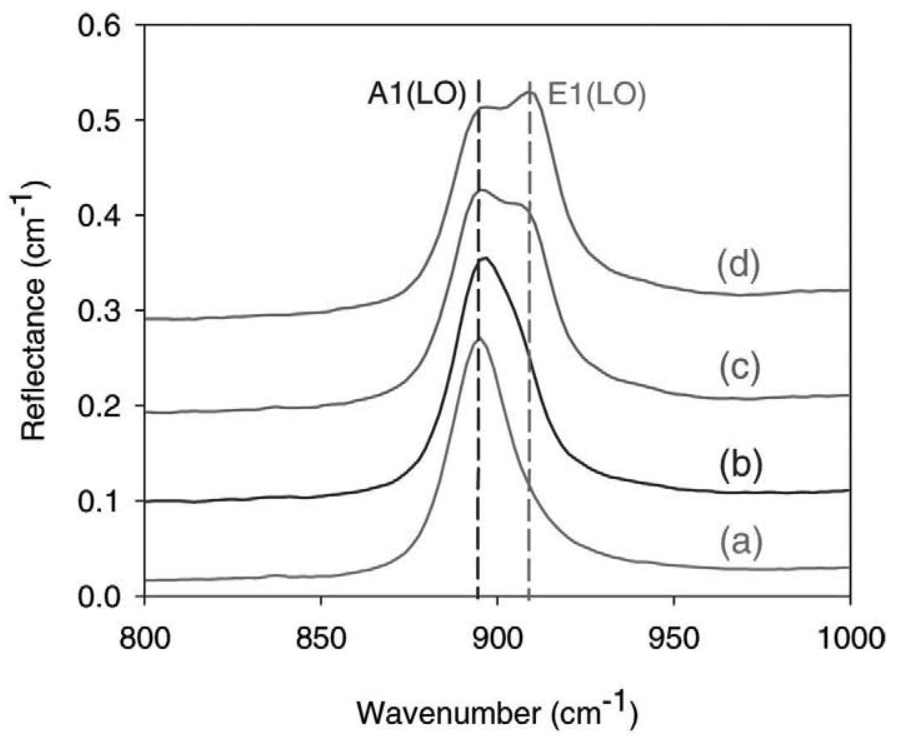

Fig. 4. Infrared absorption spectra in the reflection showing the region corresponding to the LO modes of films of Fig. 3.

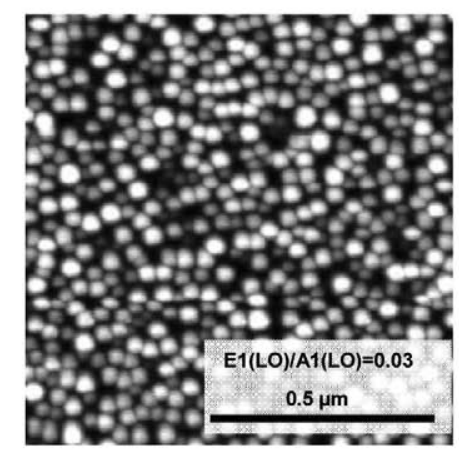

(a)

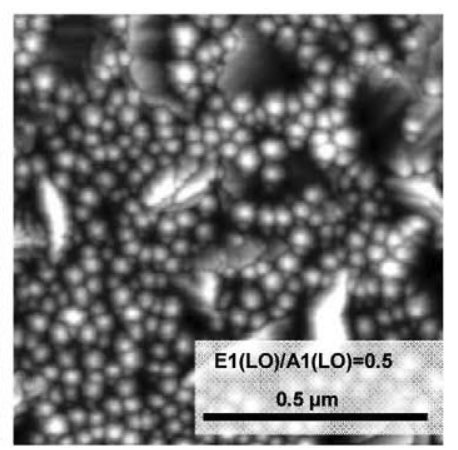

(b)
Fig. 5. Atomic force microscopy images of the surface of AlN films with different $\mathrm{E} 1(\mathrm{LO}) / \mathrm{A} 1(\mathrm{LO})$ ratios.

tionally, the E1(LO) mode [22]. Fig. 4 confirms the results indicated by XRD characterization; indeed, a gradual increase of the intensity of the $\mathrm{E} 1(\mathrm{LO})$ mode is observed as the amount of tilted grains in the samples grows. We have used the ratio of the intensities $\mathrm{E} 1(\mathrm{LO}) / \mathrm{A} 1(\mathrm{LO})$ as a quantitative measurement of the crystal quality of the samples, the crystal quality is greater for lower values of the ratio.

Fig. 5 shows the AFM images of the surface of two characteristic AIN films of different crystal quality, characterized by different $\mathrm{E} 1(\mathrm{LO}) / \mathrm{A} 1(\mathrm{LO})$ ratios. We have verified that films of thickness ranging from 0.5 to $1.5 \mu \mathrm{m}$ exhibit a similar surface, which suggests that, apart from the interfacial region corresponding to the very first stages of growth, the AlN bulk structure is very likely similar to the surface structure. It is important to note that all of the films exhibit, to a greater or lesser extent, round features corresponding to the top view of the basal plane of $(00 \cdot 2)$-oriented grains, which appear to have a uniform size (around $30 \mathrm{~nm}$ ) in the good-crystal-quality films [Fig. $5(\mathrm{a})]$. Additionally, as the $\mathrm{E} 1(\mathrm{LO}) / \mathrm{A} 1(\mathrm{LO})$ intensity ratio 
increases, faceted structures, identified as stacked tilted grains, are more and more visible at the surface of the AlN films. According to the three techniques, films with a high density of tilted grains still have an important population of $c$-axis-oriented microcrystals, although their size in the basal plane seem to be less uniform.

To investigate to what extent the polycrystalline structure of the previously described AIN films may affect the propagation of acoustic waves traveling in the directions parallel and normal to the $c$-axis, we have depicted in Fig. 6 the electromechanical coupling coefficients $k_{\mathrm{BAW}}^{2}$ and $k_{\mathrm{SAW}}^{2}$ as a function of the crystal quality of the samples, represented by the ratio $\mathrm{E} 1(\mathrm{LO}) / \mathrm{A} 1(\mathrm{LO})$.

It is clear that the two coefficients, $k_{\mathrm{BAW}}^{2}$ and $k_{\mathrm{SAW}}^{2}$, decrease dramatically as the amount of tilted grains increases, confirming that excitation and propagation of both surface and bulk waves depend significantly on the crystal quality. However, this effect seems to be more severe for the surface waves. Indeed, if we depict $k_{\mathrm{SAW}}^{2}$ as a function of $k_{\mathrm{BAW}}^{2}$ (see Fig. 7) for the films used in Fig. 6, we observe that the films with poorer texture [greater $\mathrm{E} 1(\mathrm{LO}) / \mathrm{A} 1(\mathrm{LO})$ ratios] exhibit some piezoelectric activity in the longitudinal direction but they do not possess any piezoelectric activity in the transverse direction.

The drop of the piezoelectric activity observed in BAW devices as the crystal quality worsens can be easily explained. First, it is important to note that a widening of the RC alone does not produce a significant deterioration of the piezoelectric activity, unless other defects are also present in the samples. This result can be predicted by simple geometrical considerations [23]. On the other hand, the presence of inclined grains with orientations others than $(00.2)$ is clearly linked to the reduction of the piezoelectric activity. However, the considerable drop that takes place as soon as a few inclined grains appear in the sample suggests that there are very likely other mechanisms involved in the reduction of the piezoelectric activity. Indeed, the presence of tilted grains has been associated in previous works [10] with the appearance of regions in the polycrystalline film with opposite polar orientations; so, as the amount of tilted microcrystals increases, not only is the amount of $c$-axis-oriented grains decreased, but additionally these may exhibit opposite polarity leading to a partial cancellation of the piezoelectric activity. XRD patterns of the films show that all of the films contain, to a greater or lesser extent, $c$-axis-oriented grains that are responsible for the longitudinal deformation upon the application of the electric field. Moreover, AFM images of the films surface suggest that even the films with the poorest texture may exhibit some areas free of tilted grains along the 1400-nm thickness of the film. Because it is very unlikely for the two populations of $c$-axis-oriented grains with opposite polarity to be equal, some longitudinal piezoelectric activity should be observed, as the electrical measurements reveal.

The presence of tilted grains and, hence, of inverted domains in the films also leads to the degradation of the piezoelectric activity in SAW devices. However, other

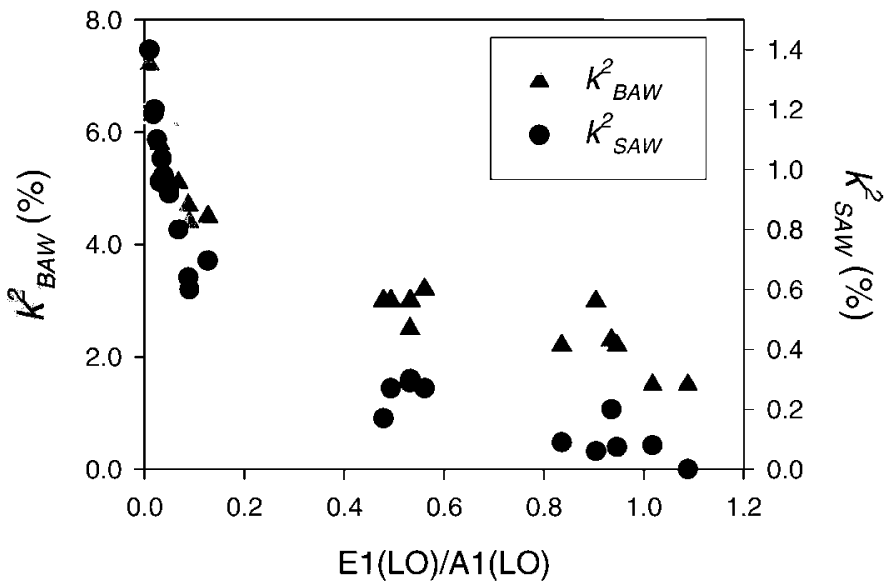

Fig. 6. Electromechanical coupling factors $k_{\mathrm{BAW}}^{2}$ (triangles) and $k_{\mathrm{SAW}}^{2}$ (circles) for different AlN films as a function of the E1(LO)/A1(LO) ratio.

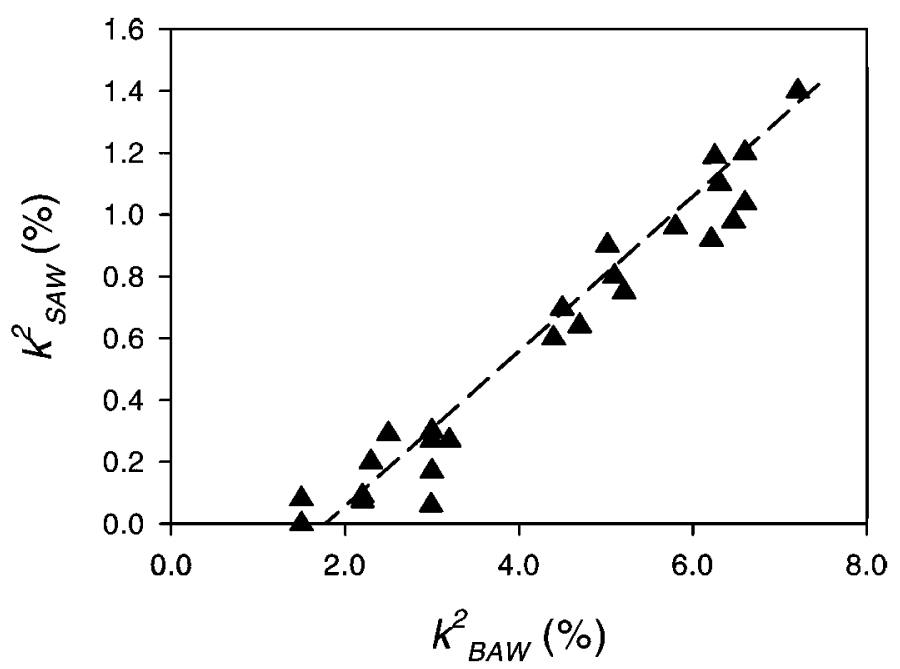

Fig. 7. as a function of $k_{\mathrm{BAW}}^{2}$ for all AIN films depicted in Fig. 6 .

mechanisms have to be taken into account here. First, one must consider that the propagation of the SAWs takes place in the transverse direction, normally to the $c$-axisoriented microcrystals. Second, we must bear in mind that the SAW propagates along a considerably large distance, compared with the thickness of the AIN layer. Therefore, the propagation of the wave requires the polycrystalline fiber-grain structure characteristic of the sputtered films to be very tightly coupled to preserve the shearing and extensional motions [24]. Previous works have shown that films of low piezoelectric quality and high propagation loss have a lower density and more loosely bound microstructure [25], which seems to be the case of our low-quality films, as suggested by AFM images. To investigate whether the crystalline defects responsible for the decrease of $k_{\mathrm{SAW}}^{2}$ also have a detrimental effect on the propagating velocity, $v_{\text {SAW }}$, we have depicted in Fig. 8 the values of $k_{\mathrm{SAW}}^{2}$ and $v_{\mathrm{SAW}}$ as a function of the crystal quality of our samples. We observe that the two magnitudes decrease simultaneously, which indicates that both excitation and propagation of surface waves are hindered by the presence 


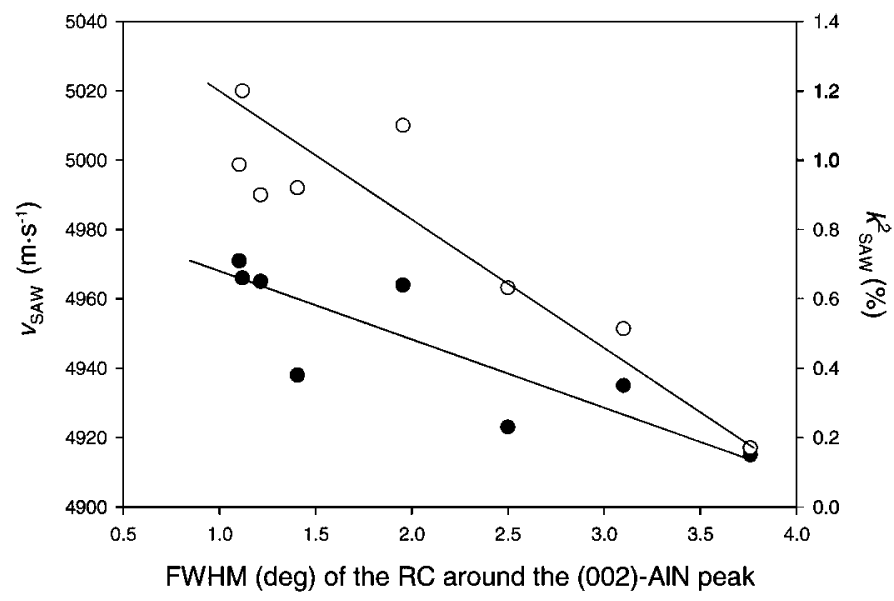

Fig. 8. Sound velocity of the surface acoustic wave $v_{\mathrm{SAW}}$ (solid circles) and $k_{\mathrm{SAW}}^{2}$ (open circles) as a function of the full-width at half-maximum (FWHM) of the rocking curves (RC) around the (00.2)-AlN reflection.

of tilted microcrystals. It is important to note that the value of $v_{\mathrm{SAW}}$, deduced from the frequency response of SAW delay lines, is a combination of the sound velocity in the AIN films and the sound velocity in the layers of the underlying substrate. Because substrate layers are kept constant for all of the SAW devices analyzed, we can attribute the observed slowing down of the surface waves to the degradation of the AIN layers. Although the reduction of the overall velocity is apparently slight, this actually corresponds to a significant variation of the velocity in the AlN film, because of the low film thickness-to-wavelength ratio used in these experiments.

\section{CONCLUSION}

The morphology, crystalline structure, and dipole orientation of AlN polycrystalline films critically affect the longitudinal and transverse excitation and propagation of acoustic waves. To efficiently excite BAWs propagating in the longitudinal thickness mode, highly c-axis-oriented AlN films with a homogeneous dipole orientation are required. The $k_{\mathrm{BAW}}^{2}$ decreases if the layers contain crystalline defects that stimulate the growth of microcrystals with different polar orientation that partially cancel the piezoelectric activity. However a total annihilation of the piezoelectric effect in the longitudinal direction cannot be achieved, because the presence of a residual population of $c$-axis-oriented grains with identical polar orientation always induces some piezoelectric activity. The influence of the misaligned grains and their grain boundaries are second-order effects in the decrease of $k_{\mathrm{BAW}}^{2}$. The requirements to excite and propagate SAWs are more demanding; not only are high-quality films necessary for the efficient excitation of the transverse waves, but additionally these films must exhibit a polycrystalline fiber-grain structure with very tightly coupled microcrystals to preserve both the shearing and extensional motions. Otherwise, the acoustic properties of the films are seriously de- graded, which can lead to the total annihilation of the piezoelectric activity in the transverse direction.

\section{REFERENCES}

[1] S. Giraud, S. Bila, M. Chatras, D. Cros, and M. Aubourg, "Bulk acoustic wave filters synthesis and optimization for multi-standard communication terminals," IEEE Trans. Ultrason. Ferroelectr. Freq. Control, vol. 57, pp. 52-58, Jan. 2010.

[2] F. Bénédic, M. B. Assouar, P. Kirsch, and D. Monéger, "Very high frequency SAW devices based on nanocrystalline diamond and aluminum nitride layered structure achieved using e-beam lithography," Diam. Relat. Mater., vol. 17, no. 4-5, pp. 804-808, Apr.-May 2008.

[3] S. González-Castilla, J. Olivares, M. Clement, E. Iborra, J. Sangrador, J. Malo, and J. I. Izpura, "Electrical detection of the mechanical resonances in AlN-actuated microbridges for mass sensing applications," Appl. Phys. Lett., vol. 92, art. no. 183506, May 2008.

[4] F. Chapuis, E. Defay, F. Casset, J. F. Manceau, F. Bastien, and M. Aï, "Microswitch with mixed piezoelectric and electrostatic actuation," in Proc. 14th Int. Solid-State Sensors, Actuators and Microsystems Conf., Lyon, France, 2007, pp. 1159-1162.

[5] C. Zuo, N. Sinha, and G. Piazza, "Very high frequency channelselect MEMS filters based on self-coupled piezoelectric AlN contourmode resonators," Sens. Actuators A, vol. 160, pp. 132-140, May 2010.

[6] V. Felmetsger, P. N. Laptev, and S. M. Tanner, "Design, operation mode, and stress control capability of S-gun magnetron for ac reactive sputtering," Surf. Coat. Tech., vol. 204, pp. 840-844, Dec. 2009.

[7] A. Ababneh, U. Schmid, J. Hernando, J. L. Sánchez-Rojas, and H. Seidel, "The influence of sputter deposition parameters on piezoelectric and mechanical properties of AlN thin films," Mater. Sci. Eng. $B$, vol. 172, pp. 253-258, Sep. 2010.

[8] R. Jakkaraju, G. Henn, C. Shearer, M. Harris, N. Rimmer, and P. Rich, "Integrated approach to electrode and AlN depositions for bulk acoustic wave (BAW) devices," Microelectron. Eng., vol. 70, no. $2-4$, pp. 566-570, Nov. 2003.

9] J. S. Cherng and D. S. Chang, "Effects of outgassing on the reactive sputtering of piezoelectric AlN thin films," Thin Solid Films, vol. 516, pp. 5292-5295, Jun. 2008.

[10] J. Olivares, S. González-Castilla, M. Clement, A. Sanz-Hervás, L. Vergara, J. Sangrador, and E. Iborra, "Combined assessment of piezoelectric AlN films using X-ray diffraction, infrared absorption and atomic force microscopy," Diam. Relat. Mater., vol. 16, no. 4-7, pp. 1421-1424, Apr.-Jul. 2007.

[11] T. Kamohara, M. Akiyama, N. Ueno, M. Sakamoto, N. Kuwano, K. Kano, A. Teshigahara, and N. Kawahara, "Influence of sputtering pressure on polarity distribution of aluminium nitride thin films," Appl. Phys. Lett., vol. 89, no. 4, art. no. 243507, Dec. 2006.

[12] Q.-B. Zhou, L. Yue-kai, and S.-Y. Zhang, "Extraction of electromechanical coupling coefficient of piezoelectric thin films deposited on substrates," Ultrasonics, vol. 39, no. 5, pp. 377-382, Aug. 2001.

[13] Q. Chen and Q. M. Wang, "The effective electromechanical coupling coefficient of piezoelectric thin-film resonators," Appl. Phys. Lett., vol. 86, art. no. 022904, 2005.

[14] J. F. Rosenbaum, Bulk Acoustic Wave Theory and Devices. Boston, MA: Artech House, 1988.

[15] C. K. Campbell, Surface Acoustic Wave Devices for Mobile and Wireless Communications. San Diego, CA: Academic, 1998.

[16] E. Iborra, L. Vergara, J. Sangrador, M. Clement, A. Sanz-Hervás, and J. Olivares, "Circuital model for the analysis of the piezoelectric response of AlN films using SAW filters," IEEE Trans. Ultrason. Ferroelectr. Freq. Control, vol. 54, no. 11, pp. 2367-2375, Dec. 2007.

[17] S. Trolier-Mckinstry and P. Muralt, "Thin film piezoelectrics for MEMS," J. Electroceram., vol. 12, no. 1-2, pp. 7-17, 2004.

[18] F. Hickernell, "Zinc oxide films for acoustoelectric device applications," IEEE Trans. Sonics Ultrason., vol. 32, no. 5, pp. 621-629, Sep. 1985.

[19] R. S. Wagers and G. S. Kino, "Analysis of the electric potential of an acoustic surface wave propagating on a layered half space," IEEE Trans. Sonics Ultrason., vol. SU-21, pp. 209-213, Jul. 1974.

[20] L. E. MeNeil, M. Grimsditch, and R. H. French, "Vibrational spectroscopy of aluminum nitride," J. Am. Ceram. Soc., vol. 76, no. 5, pp. 1152-1156, 1993. 
[21] M. F. MacMillan, R. P. Devaty, and W. J. Choyke, "Infrared reflectance of thin aluminum nitride films on various substrates," Appl. Phys. Lett., vol. 62, no. 7, pp. 750-752, 1993.

[22] E. Iborra, M. Clement, L. Vergara, A. Sanz-Hervás, J. Olivares, and J. Sangrador, "Dependence of the IR reflectance LO absorption bands on the crystalline texture of AlN films," Appl. Phys. Lett., vol. 88, art. no. 231901, Jun. 2006.

23] S. Mishin, D. R. Marx, B. Sylvia, V. Lughi, K. L. Turner, and D. R. Clarke, in Proc. 2003 IEEE Int. Ultrasonics Symp., Honolulu, HI, pp. $2028-2032$.

24] K. Hashimoto, T. Omori, and M. Yamaguchi, "Requirements for piezoelectric thin film applications to radio frequency acoustic wave devices," Ferroelectrics, vol. 380, pp. 73-80, Dec. 2008.

[25] S. H. Kim, K. H. Lee, D.-J. Kim, and Y. S. Yoon, "Frequency responses of an AlN/IDT/Si surface acoustic wave device based on AlN thin films with different grain sizes," J. Ceram. Process. Res., vol. 8 , no. 2 , pp. $125-128,2007$.

Marta Clement received the M.S. degree in physics in 1985 from the Universidad Complutense de Madrid, Spain, and the Ph.D. degree in physics in 1990 from the Universidad Autónoma de Madrid, Spain. She spent a year at AT\&T Microelectronics Spain as process engineer in the plasma area. In 1991, she joined the Department of Electronic Technology at the Universidad Politécnica de Madrid as associate professor, where she initially focused his research on the development of electronic materials for infrared sensor applications. She is a charter member of the GMME (Group of Microsystems and Electronic Materials) from the same university, where she has carried out her research since 1990 on the development of thin-film materials and technologies for SAW, BAW, and MEMS devices. In 2011, the members of the GMME joined the CEMDATIC (Center of Advanced Materials and Devices for IST) from the same university.

Jimena Olivares received the B.Sc. degree and the M.Sc. degree in physics from the Universidad Autónoma de Madrid, Spain, in 1995 and 1997, respectively. She received the Ph.D. degree in physics from the Universidad Politécnica de Madrid, Spain, in 2001 for research work on polycrystalline SiGe thin films. She spent two years in a Spanish com- pany, working as an expert in technology transfer and research projects management. In 2003, she joined the GMME of the Universidad Politécnica de Madrid and, in 2011, CEMDATIC. Her current research interests are the development of functional acoustic materials (piezoelectric, insulating and conducting materials of high/low acoustic impedance) and the development of technologies for SAW, BAW, and MEMS devices.

Jose Capilla received the M.S. degree in telecommunication engineering from the Universidad Politécnica de Madrid, Spain, in 2009. His degree work was based on pulsed-de AlN sputtered films. He joined the GMME of the Universidad Politécnica de Madrid in 2009. The same year, he started his Ph.D. research on thin-film resonators and filters based on AlN and insulating materials for their application in acoustic mirrors, which are his current research interests.

Jesús Sangrador received the $\mathrm{Ph} . \mathrm{D}$. degree in telecommunication engineering from the Universidad Politécnica de Madrid, Spain, in 1982 for his research on the fabrication and characterization of silicon solar cells. In 1982, he joined the Department of Electronic Technology of the same university as an associate professor and initially focused his research on the development of infrared detectors based on MCT and SiGe compounds. Since 1990, he has been a full professor at this university. His current research interest areas are material deposition and thin-film technology for sensor applications.

Enrique Iborra received the M.Sc. degree in physics from the Universidad Complutense de Madrid, Spain, in 1982. He received the Ph.D. degree (with distinction) in physics from the same university in 1986. In 1987 , he joined the department of electronic technology at the Universidad Politécnica de Madrid as associate professor. In 1995, he founded the Group of Microsystems and Electronic Materials (GMME) at the Universidad Politécnica de Madrid and has been the head of the group since then. In 2009, he became a full professor at the same university. He has been working in thin-film electronic materials and sensor devices since 1982. His current research interests are the applications of piezoelectric AlN thin films to SAW, BAW, and RF-MEMS devices. 